\title{
High Energy Physics
}

The series of articles and discussions on elementary particle physics organized by J. Meyer of Brazil (and formerly of CERN, Geneva) was perhaps a little outside the main theme of the Bucharest Conference but the subject is in such a lively state it could hardly be ignored. The range of topics covered was wide but a considerable amount of unity could be noted.

In November 1974 Brookhaven and Stanford published the discovery of a very narrow resonance at $3.1 \mathrm{GeV}$ invariant mass in the $\mathrm{e}^{+} \mathrm{e}^{-}$system. This and a very long list of experiments carried out since - one can mention also those at Frascati, DESY, Cornell (photoproduction), Fermilab (production by photons and neutrons) and CERN (ISR energies) - makes "new particles" the hottest news in high energy and particle physics. G. Capon, C. Rubbia and J. Bell gave excellent descriptions of the excitement that has been generated which is by no means confined to one single aspect. Let us start by having a look at the first experiments.

At Brookhaven they were studying the reaction $\mathrm{p}+\mathrm{p} \rightarrow \mathrm{e}^{+}+\mathrm{e}^{-}+$anything.

The momenta of the $\mathrm{e}^{+}$and the $\mathrm{e}^{-}$ were measured with high precision in a double spectrometer. At Stanford they examined the reaction $\mathrm{e}^{+}+\mathrm{e}^{-}$ $\rightarrow$ anything in the SPEAR storage ring. The study of the $e^{+} e^{-}$system is of considerable interest for our understanding of photon-like objects such as the vector mesons $\varrho, \omega, \varphi, \varrho^{\prime}$ and also for the search for more states of a similar nature. Furthermore a comparison of the cross-sections of the reactions :

$\mathrm{e}^{+}+\mathrm{e}^{-} \rightarrow \mu^{+}+\mu^{-}$and $\mathrm{e}^{+}+\mathrm{e}^{-} \rightarrow$ hadrons as a function of centre of mass energy squared, s (and in particular for large s) yields :

$$
R(s)=\sigma_{\text {had }}(s) / \sigma_{\mu \mu}(s)
$$

which in parton models measures the sum of the squares of the parton charges.

$$
R(s)=\sum_{n=1}^{N} Q_{n}^{2}
$$

This holds for models with $\mathrm{N}$ different types of spin $1 / 2$ partons which are point-like and which cannot exist in a free state i.e. they always go into a hadronic final state. As an example, the ordinary quark model with quarkcharges $+2 / 3,-1 / 3$ and $-1 / 3$ gives $R=2 / 3$. So there were very good and precise motivations for both the Brookhaven and the Stanford experiments. The surprise was that the $\mathrm{e}^{+} \mathrm{e}^{-}$system showed a very narrow resonance at $3.095 \pm .004 \mathrm{GeV}$ as illustrated by the Stanford results in Fig. 1. We interpret this as the existence of a relatively long-lived particle so that the process is

$\mathrm{e}^{+}+\mathrm{e}^{-} \rightarrow \psi \rightarrow$ hadrons

$$
\begin{aligned}
& \longrightarrow \mathrm{e}^{+}+\mathrm{e}^{-} \\
& \mu^{+}+\mu^{-}
\end{aligned}
$$

where "hadrons" cover a very long list of decay modes.

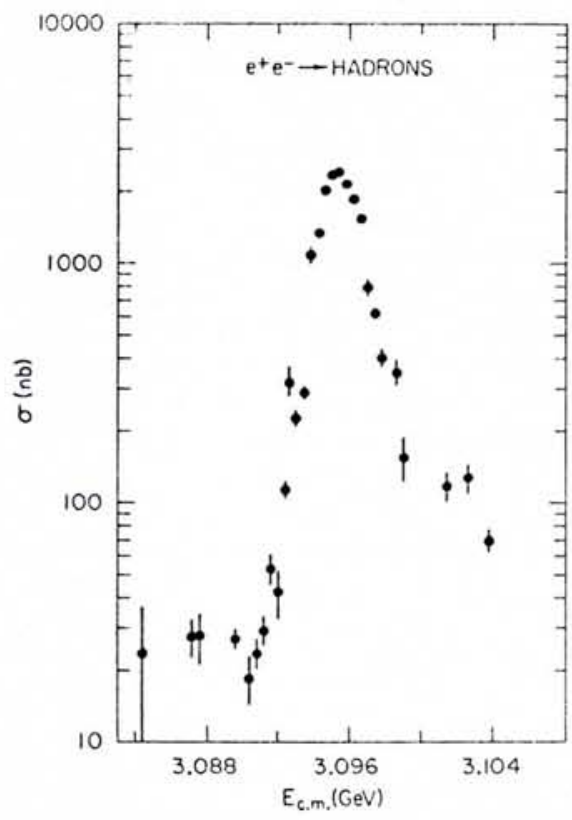

Fig. 1. - The total cross-section for $e^{+}+e^{-}-$ hadrons in the neighbourhood of $3.1 \mathrm{GeV}$ centre of mass energy. Data from the review of G.J. Feldman and M.L. Perl, Physics Reports 19C, No. 5, 1975.

Since the first experiments at Brookhaven and Stanford an almost incredible amount of experimental material has been accumulated. First of all we now know about the existence of many heavy particles. The second was an object with mass $\cong 3.7 \mathrm{GeV}$ which has been given the name $\psi^{\prime}$. Its principal decay has been spectacular and is reconstructed in Fig. 2. The basic idea of the decay is :

$$
\begin{aligned}
\psi^{\prime} \rightarrow \psi+\pi^{+}+\pi^{-} & \\
& \rightarrow e^{+}+e^{-}
\end{aligned}
$$

Fig. 3 shows the level scheme of the new particles as predicted by the quark model including the special quantum number called charm. A quark with charm combines with one with anti-charm to the spectroscopic states indicated in Fig. 3. There are now seven or eight states known and decay modes are measured in detail. For the $\psi$ one has observed close to 20 different decay modes. However, the quarks are not seen and so far, charmed mesons have not been found.

Rubbia mentioned that there was evidence from Stanford of the existence of a heavy lepton. If confirmed, this will be the sensation for the next conference.

Rubbia's talk was entitled "Still higher energies: why?". With the idea in mind that the heavy intermediate boson responsible for $\beta$ decay has a mass which is likely to be of the order of $100 \mathrm{GeV}$ the "why?" is not difficult to understand. V.P. Sarantzev answered the question "how?". The answer is essentially by brute force. $\mathrm{He}$ didn't tell us however where the money would come from.

Bell's lecture on "Perspectives in unified field theory: Unified gauge theories of weak, electromagnetic (+ strong ?) interactions" needs to be studied in full. He reminded us of the fact that it is gauge invariance which makes QED renormalizable and showed how similar ideas can be introduced in weak interactions. There are complications and new particles are necessary. It is indeed interesting that the resulting expressions have the strong interaction coupling constant in a denominator - thus hope for a complete unification. He went through our theoretical picture (leading among others to the ' $\psi^{\prime}$ scheme of Fig. 3)

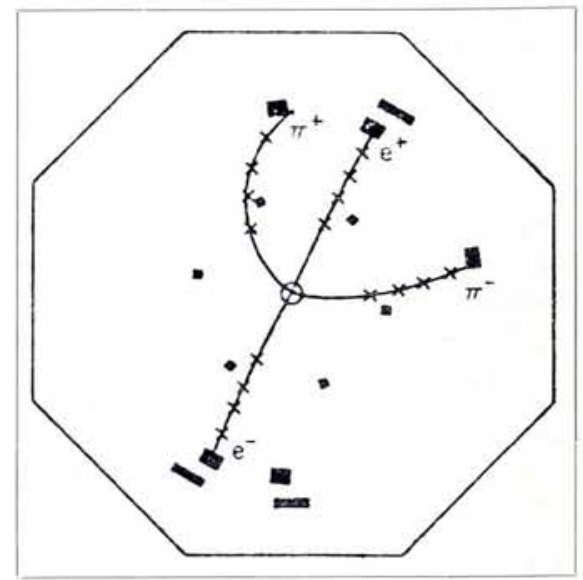

Fig. 2. - A computer reconstruction of the decay $\psi^{\prime} \rightarrow \psi x \pi$ where $\psi^{\prime} \rightarrow e^{+} e^{-}$from the SLAC-LBL magnetic detector at SPEAR. The event is seen in the $x-y$ projection where $z$ is the beam and magnetic field direction. The closed rectangles represent trigger and shower counters which fired. From the review of G.J. Feldman and M.L. Perl, Physics Reports 19C, No. 5, 1975. 
and introduced quarks, charm etc. $\mathrm{He}$ discussed many models and finished by illustrating how the basic ideas are quite unifying for physics as such, since most of them have been used before.

D.H. Wilkinson, gave a lecture entitled "Elementary particles and nuclei" in which he presented a strong case for nuclear physics as an important tool in the investigation of elementary particles. He did so by talking about two sets of $\beta$ - decay experiments only and succeeded thereby in giving an excellent background setting for the following speaker (Bell). His first argument was based on conservation of the vector current, on the $\mu-e$ universality in the Cabibbo sense and on the Salam-Weinberg unification of weak and electromagnetic interactions. The experimental data are the $\mathrm{ft}$ values for $\mathrm{O}^{+} \rightarrow 0^{+}$superallowed $\beta$ - transitions, the muon $\mathrm{ft}$ value and the Cabibbo angle. An estimate of the mass of the neutral boson obtained from neutral current investigations is also necessary. Putting all this together one obtains a value for the average electric charge of the quarks. The result is: $\mathrm{Q}=(.20 \pm .10) \mathrm{e}$ in agreement with $1 / 2(2 / 3-1 / 3)=.167$. His second result was a limit on the strength of possible second class currents in the Fermi interaction by $\mathrm{ft}$ value comparison for $\beta^{+}$and $\beta^{-}$mirror transitions inside the same isospin multiplet. The result is again as precise as the best high energy result ( $\Sigma$ - meson decay).

In addition nuclear physics had its own sessions organized by $\mathrm{M}$. Petrascu (Bucharest). A.M. Baldin gave a plenary session talk on heavy ion physics with a good resume of one very important line of research in Dubna and with a repeated invitation for others to come and participate. The main speakers in the afternoon sessions were Z. Szymanski who talked about the possible existence of new elements or of exotic states of atomic nuclei, and S.G. Nilsson who gave a lecture on the recently discovered high angular momentum states and the types of effective optical potentials which are needed for the description of such states. There were also lectures by W. Greiner and $H$. Bethge on heavy ion physics, the first from the point of view of nuclear physics including shock waves in nuclear matter and the second from the point of view also of technological applications.

O.K.-H

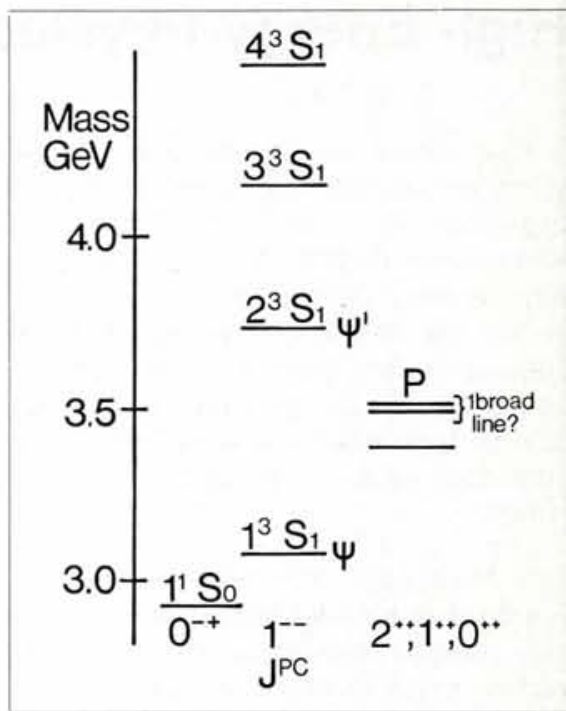

Fig. 3. - The level scheme of the new particles as predicted by the quark model including the special quantum number called charm.

\section{Energy Strategies}

The three plenary lectures, directly related to Energy Strategies were given by Haefele (Laxenburg) on "Energy Strategies", Weinberg (Oak Ridge) on "The Maturity of Nuclear Energy", Braams (Jutphaas) on "State of Thermonuclear Research" while the speech by Ursu on "Energy Today: New Goals and Challenges for Physics" was mainly directed at the present knowledge and more local situations. The lectures of the plenary sessions will be published in the proceedings of the conference, therefore it seems appropriate to give in this short report a somewhat detailed review of the subjects discussed in the two afternoon panel sessions.

It is obvious that sufficient food and energy are the two primary requirements mankind is facing. In those regions of the world where large populations are living due to acceptable climatic conditions sufficient arable land is also available, otherwise no population growth would have taken place. Today it is possible to live in many parts of the world with an even larger population and still produce sufficient food if sufficient fertiliser (nitrogen) is available. This means that energy is needed for the production of fertiliser. Furthermore the land has to be ploughed. This requires energy for the production of the construction materials of the tractors anid energy for their operation. The rest is done by photosynthesis. For the provision of reasonable housing and transport the same is true. In the lecture by Haefele many such relations were shown.

In different parts of the world the local energy supply possibilities as well as the energy requirements determine the energy policies. The attitude of the USA with its still reasonably large energy resources with respect to coal, oil and gas as well as nuclear energy tends to be as far as possible independent of outside supplies.

In Western Europe such a possibility does not exist hence it seems very difficult to come to one common energy strategy.
In the first afternoon meeting of the two panel sessions, Weinberg (Oak Ridge) and Hemans (Arnhem) introduced short surveys on Energy Research for the year 2000 and in relation to this long term goal on the development of thermal breeders. It was interesting that both lecturers had the opinion that thermal breeding either with a molten salt system or with an aqueous homogeneous suspension system, should receive more support and stimulation, realizing the importance of thermal breeding next to fast breeding. It should be of interest for a group of experts in this field to review the possibilities and perhaps recommend what to do or not to do.

Two international organizations Unesco (Berkovsky) and the OECD (Drilhon) also presented introductions about their activities in the field. It is of real importance that such international organizations are presenting a platform for mutual discussion among all interested countries in the world. Also the second world wide 